INFN-NA-IV-93/20

DSF-T-93/20

\title{
Quantum Limits in Interferometric GW Antennas in the Presence of Even and Odd coherent states
}

\author{
Nadeem A. Ansari, L. Di Fiore, M. A. Man'ko*, V.I. Man'ko*, S. Solimeno \\ and F. Zaccaria \\ Dipartimento di Scienze Fisiche, Universita di Napoli "Federico II" \\ Istituto Nazionale di Fisica Nucleare, Sezione di Napoli Mostra d'Oltremare, \\ Pad.20, 80125 Napoli, Italy
}

1* On leave from Lebedev Physics Institute, Moscow, Russia. 


\begin{abstract}
We discuss a model for interferometric GW antennas without dissipative or active elements. It is predicted that the even and odd coherent states may play an alternative role to squeezed vacuum states in reducing the optimal power of the input laser.
\end{abstract}


The problem of detecting gravitational waves has been a subject of interest since last many years [1]. Specifically, the quantum sensitivity of Michelson interferometric gravitational wave detection has been described in detail by Caves [2]. An important ingredient improving the sensitivity of such detectors (GWD) is using the appropriate states of light beam through the two ports of the Michelson interferometer. Caves [2] showed, in fact, that if one uses coherent light [3] from the first port of the interferometer, then the optimal sensitivity is limited by the vacuum fluctuations which enter through the unused port of the interferometer. In such set up the lower limit on the optimal power of the input laser comes out to be quite large and of no experimental interest. Caves [2] suggested to reduce considerably the above limit by squeezing the vacuum field entering through the unused port [4].

The main purpose of this communication is to answer the following question: "are there any other nonclassical states different from squeezed states, which can replace squeezed vacuum in GWD for a better quantum sensitivity of the Michelson interferometer"? We predict a possible positive answer to this question in the use of even or odd coherent states [5]. Even coherent states are closely related to the squeezed vacuum states because they too are superposition of even number states, but with different coefficients. Different nonclassical properties of even coherent states and theoretical predictions for their possible generation have been discussed in detail in Refs.(6-11).

In the Michelson interferometer (Fig.1) we have two incoming fields through ports $P_{i}, i=1,2$, described by the operators $\left(a_{i}, a_{i}^{\dagger}\right)$ acting on a Hilbert space $\mathcal{H}^{a}=\mathcal{H}_{1}^{a} \otimes \mathcal{H}_{2}^{a}$. To them corresponds two fields at the mirrors $M_{i}$ described by $\left(b_{i}, b_{i}^{\dagger}\right)$ acting on $\mathcal{H}^{b}=\mathcal{H}_{1}^{b} \otimes \mathcal{H}_{2}^{b}$ and two outgoing fields at $P_{i}$ described by $\left(c_{i}, c_{i}^{\dagger}\right)$ on $\mathcal{H}^{c}=\mathcal{H}_{1}^{c} \otimes \mathcal{H}_{2}^{c}$. The basis in $\mathcal{H}_{i}^{\rho}, \rho=a, b, c$, will be denoted $\left\{\mid n, i, \rho>, \quad n \in Z^{+}\right\}$. We simplify the Michelson interferometer as a device with two arms at the end of which two outer mirrors $M_{i}$ are attached to some string, thus behaving as two pendula, without considering Fabry-Perot cavities and beam delaying optics into these arms. We will suppose that in all processes the dissipative and active effects are negligible so that conservation of energy in ensured. The Hamiltonian in $\mathcal{H}^{\rho}$ is taken to be

$$
H^{\rho}=\hbar \omega\left(\rho_{1}^{\dagger} \rho_{1}+\rho_{2}^{\dagger} \rho_{2}\right)
$$

with $\omega$ the frequency and $\hbar$ the Planck's constant. Implicit here is the assumption of equal frequencies for the mode 1 and 2. This can be achieved by introducing some degree of interaction among the two modes, which anyhow can be ignored in a first approximation, as in Ref.[2]. All $\mathcal{H}^{\rho}$ are unitarily equivalent and the operators $H^{\rho}$ are connected each other by $2 \times 2$ unitary matrices, the elements of which depend on the physical and geometrical parameters of the interferometer. For instance, we will write

$$
b=V a, \quad b^{\dagger}=a^{\dagger} V^{\dagger}
$$


where

$$
\begin{aligned}
a & =\left(\begin{array}{l}
a_{1} \\
a_{2}
\end{array}\right) ; \quad b=\left(\begin{array}{c}
b_{1} \\
b_{2}
\end{array}\right) \\
a^{\dagger} & =\left(\begin{array}{ll}
a_{1}^{\dagger} & a_{2}^{\dagger}
\end{array}\right) ; b^{\dagger}=\left(\begin{array}{ll}
b_{1}^{\dagger} & b_{2}^{\dagger}
\end{array}\right)
\end{aligned}
$$

and $V \in U(2)$ group. We conveniently write

$$
V=\Phi K
$$

with

$$
\begin{aligned}
\Phi & =\left(\begin{array}{cc}
e^{i \phi_{1}} & 0 \\
0 & e^{i \phi_{2}}
\end{array}\right) \\
K & =\left(\begin{array}{cc}
\alpha_{1} & \beta_{2} \\
\beta_{1} & \alpha_{2}
\end{array}\right)
\end{aligned}
$$

In the above $\alpha_{i}$ and $\beta_{i}$ are the complex transmittivity and reflectivity parameters of the beam splitter (BS) arbitrarily oriented for the ith input field mode respectively and $\phi_{i}$ is the phase distance between $\mathrm{BS}$ and the mirror $M_{i}$. Also

$$
c=U a ; c^{\dagger}=a^{\dagger} U^{\dagger}
$$

and

$$
U=-K^{T} \Phi^{2} K=-V^{T} V
$$

The presence of negative sign in the above equation is due to the phase change on reflections at the mirrors.

Following [2], we have two sources of errors which set the lower quantum limit $\Delta z$ on the sensitivity $\mathrm{z}$ of GW antennas: (i) radiation pressure on $M_{i}$ and (ii) photon counting noise due to the fluctuations in the number of photons in the input fields

$$
\Delta z=\sqrt{\left(\Delta z_{r p}\right)^{2}+\left(\Delta z_{p c}\right)^{2}}
$$

In this

$$
\left(\Delta z_{r p}\right)^{2}=\sigma_{r p}^{2}(\hbar \omega \tau / m c)^{2}
$$

where

$$
\sigma_{r p}^{2}=<\left(b^{\dagger} \sigma_{3} b\right)^{2}>-<b^{\dagger} \sigma_{3} b>^{2}
$$

and

$$
\left(\Delta z_{p c}\right)^{2}=\sigma_{p c}^{2}\left(\frac{\partial<c^{\dagger} \sigma_{3} c>}{\partial\left(\phi_{2}-\phi_{1}\right)}\right)^{-2}
$$

where

$$
\sigma_{p c}^{2}=<\left(c^{\dagger} \sigma_{3} c\right)^{2}>-<c^{\dagger} \sigma_{3} c>^{2}
$$


In the above, $\tau$ is the observation time and $m$ the mass of each end mirrors. Here we consider fixed BS as in Ref.(2). After little algebra we can write

$$
\begin{aligned}
\sigma_{r p}^{2} & =\left(U^{\dagger} \sigma_{3} U\right)_{i k}\left(U^{\dagger} \sigma_{3} U\right)_{m n} T_{i k m n} \\
\sigma_{p c}^{2} & =\left(V^{\dagger} \sigma_{3} V\right)_{i k}\left(V^{\dagger} \sigma_{3} V\right)_{m n} T_{i k m n}
\end{aligned}
$$

with the summation over repeated indices taken from 1 to 2 and

$$
T_{i k m n}=<a_{i}^{\dagger} a_{k} a_{m}^{\dagger} a_{n}>-<a_{i}^{\dagger} a_{k}><a_{m}^{\dagger} a_{n}>
$$

This allows an easy comparison between situations arising from the use of different types of input fields.

Combining Eqs.(8-13) yields

$$
(\Delta z)^{2}=X_{i k m n} T_{i k m n} \quad(i k m n=1,2)
$$

where $X_{i k m n}$ contain the geometrical and physical properties of the antenna while the second factors $T_{i k m n}$ depend only on the incoming fields.

For a simple Michelson interferometer, Caves suggested to use squeezed vacuum light in order to minimize the input laser power [2]. Equation (15) permits to investigate very general states of the input field like even and odd coherent states, correlated states, states with higher order squeezing, etc. In particular, we will illustrate in this communication the dependence of the optimal $\Delta z$ on the characteristic parameters of the even or odd coherent states from the second port of interferometer.

First, we will evaluate the matrix $X_{i k m n}$ as far as the geometrical and physical parameters of the Michelson interferometer are concerned.

If we consider a 50-50\% BS, then the elements of the matrix $K$ are

$$
\begin{aligned}
\alpha_{1}=\alpha_{2} & =\frac{e^{i \delta}}{\sqrt{2}} \\
\beta_{1}=\beta_{2} & =\frac{e^{i \mu}}{\sqrt{2}}
\end{aligned}
$$

where $\delta$ is the phase because of the BS which can be set to zero for an ideally thin $\mathrm{BS}$ while $\mu$ is the phase introduced by the BS between the reflected and transmitted waves and for simplicity we take $\mu=\pi / 2$. Then

$$
V^{\dagger} \sigma_{3} V=\left(\begin{array}{cc}
0 & i \\
-i & 0
\end{array}\right)
$$

and

$$
U^{\dagger} \sigma_{3} U=\left(\begin{array}{ll}
-\cos \phi & -\sin \phi \\
-\sin \phi & \cos \phi
\end{array}\right)
$$


in $\phi=2\left(\phi_{2}-\phi_{1}\right)$. Also

$$
<c^{\dagger} \sigma_{3} c>=<a^{\dagger} \sigma_{3} a>\cos \phi
$$

If the interferometer has to operate in a dark fringe then the arms lengths can be adjusted to have $\phi=\frac{(2 n+1) \pi}{2}$ and dark fringes correspond to the situation where no field contributions is present into the difference of the output photon numbers. In such cases we have

$$
U^{\dagger} \sigma_{3} U=\left(\begin{array}{cl}
0 & -1 \\
-1 & 0
\end{array}\right)
$$

Then $X_{i k m n}$ become

$$
\begin{aligned}
& X_{1212}=X_{2121}=-A^{2}+B^{2} \\
& X_{1221}=X_{2112}=A^{2}+B^{2}
\end{aligned}
$$

where

$$
\begin{aligned}
A & =\left(\frac{\hbar \omega \tau}{m c}\right) \\
B & =\left(\frac{\partial I}{\partial Z}\right)^{(-1)}
\end{aligned}
$$

and

$$
\begin{aligned}
I & =\left\langle c^{\dagger} \sigma_{3} c\right\rangle \\
Z & =\phi \frac{c}{2 \omega}
\end{aligned}
$$

The variable $\mathrm{Z}$ corresponds to the difference between the displacements of the two outer mirrors caused by the radiation pressure with respect to their mean positions in the absence of any field.

We will now evaluate the factors $T_{i k m n}$ in (i) Caves setup and (ii) in a new one which replaces the squeezed light with even or odd coherent light.

In order to evaluate the contribution of the fields which are applied to the two ports of the interferometer for GW detection, we will assume a coherent light for the field relative to port one of the interferometer while for the second port we will consider the two situations (i) by squeezing the vacuum fluctuations (the situation considered by the Caves[2]) and (ii) applying even or odd coherent states. We will show the important role played by these states in order to get a better detection sensitivity and to reduce the optimal input laser power.

When coherent laser light from port one and squeezed vacuum from the other port of the interferometer are applied, the two fields are anticorrelated. The states of $\mathcal{H}^{a}$ can be written as

$$
\left|\psi>=\mathcal{D}_{1}(\alpha)\right| 0,1, a>e^{\frac{\xi a_{2}^{\dagger 2}-\xi^{*} a_{2}^{2}}{2}} \mid 0,2, a>
$$


where $\mathcal{D}_{i}(\alpha)=e^{\left(\alpha a_{i}^{\dagger}-\alpha^{*} a_{i}\right)} \mathrm{i}=1,2, \alpha \in C$, and $\xi=r e^{i \theta_{1}}$. It is easy to see that in such states $<a_{1} a_{2}>,<a_{1}^{\dagger} a_{2}>$, etc., are equal to zero.

If we take $\alpha$ to be real for simplicity then we have

$$
\begin{aligned}
& T_{1111}=\alpha^{2} \\
& T_{1122}=0 \\
& T_{1212}=-\alpha^{2} \sinh r \cosh r e^{i \theta_{1}} \\
& T_{1221}=\alpha^{2} \sinh ^{2} r+\alpha^{2} \\
& T_{2112}=\alpha^{2} \sinh ^{2} r+\sinh ^{2} r \\
& T_{2121}=-\alpha^{2} \sinh r \cosh e^{-i \theta_{1}} \\
& T_{2211}=0 \\
& T_{2222}=2 \sinh ^{2} r
\end{aligned}
$$

When even or odd coherent states replace squeezed vacuum in port two the states of $\mathcal{H}^{a}$ to be taken into account are

$$
\begin{aligned}
\mid \psi> & =\mid \alpha, \beta_{ \pm}> \\
& =\mathcal{D}_{1}(\alpha) \mid 0,1, a>N_{ \pm}\left(\mathcal{D}_{2}(\beta) \pm \mathcal{D}_{2}(-\beta) \mid 0,2, a>\right.
\end{aligned}
$$

where ',+- ' signs correspond to even and odd coherent states respectively and their normalization constants are

$$
\begin{aligned}
& N_{+}=\frac{1}{2 e^{-\frac{|\beta|^{2}}{2}} \sqrt{\cosh |\beta|^{2}}} \\
& N_{-}=\frac{1}{2 e^{-\frac{|\beta|^{2}}{2}} \sqrt{\sinh |\beta|^{2}}}
\end{aligned}
$$

For the even light, coefficients $T_{i k m n}$ take the following values

$$
\begin{aligned}
& T_{1111}=\alpha^{2} \\
& T_{1122}=0 \\
& T_{1212}=\alpha^{2}|\beta|^{2} e^{2 i \theta_{2}} \\
& T_{1221}=\alpha^{2}|\beta|^{2} \tanh |\beta|^{2}+\alpha^{2} \\
& T_{2112}=\alpha^{2}|\beta|^{2} \tanh |\beta|^{2}+|\beta|^{2} \tanh |\beta|^{2} \\
& T_{2121}=\alpha^{2}|\beta|^{2} e^{-2 i \theta_{2}} \\
& T_{2211}=0 \\
& T_{2222}=|\beta|^{4}-|\beta|^{4} \tanh ^{2}|\beta|^{2}+|\beta|^{2} \tanh |\beta|^{2}
\end{aligned}
$$

in which $\theta_{2}$ is the phase of $\beta$.

For odd coherent states we get the same expressions as above, except that $\tanh |\beta|^{2}$ should be replaced by $\operatorname{coth}|\beta|^{2}$. 
The general expression for $(\Delta z)^{2}$, irrespective of the nature of the incoming fields can now be written as

$$
\left(\Delta z^{2}\right)=A^{2}\left(T_{1221}+T_{2112}-T_{1212}-T_{2121}\right)+B^{2}\left(T_{1221}+T_{2112}+T_{1212}+T_{2121}\right)
$$

This quantity depends on the incoming field through $P_{1}$ and we denot by $\left(\alpha_{o p t}^{2}\right)^{(o)}=m c^{2} /(2 \hbar \omega \tau)$ the intensity of this field which minimizes $(\Delta z)^{2}$ when at $P_{2}$ the ordinary vacuum is present. Then, it can be seen that the value which minimizes $(\Delta z)^{2}$, which we call $\left(\alpha_{o p t}^{2}\right)^{(s q)}$, in presence of squeezed vacuum at $P_{2}$, under the condition $\alpha^{2}>>\sinh ^{2} r$ and $\theta_{1}=0$, is

$$
\left(\alpha_{o p t}^{2}\right)^{(s q)}=e^{-2 r}\left(\alpha_{o p t}^{2}\right)^{(o)}
$$

This is the Caves result, which allows to reduce the intensity of the input laser beam to values experimentally significant.

The analogous analysis for the cases of even or odd coherent states replacing squeezed vacuum, under the condition that $\alpha^{2}>>|\beta|^{2} \tanh |\beta|^{2}$ gives

$$
\left(\alpha_{o p t}^{2}\right)^{(e v)}=\sqrt{\frac{2|\beta|^{2} \tanh |\beta|^{2}+2|\beta|^{2} \cos 2 \theta+1}{2|\beta|^{2} \tanh |\beta|^{2}-2|\beta|^{2} \cos 2 \theta+1}}\left(\alpha_{o p t}^{2}\right)^{(o)}
$$

and

$$
\left(\alpha_{\text {opt }}^{2}\right)^{(\text {odd })}=\sqrt{\frac{2|\beta|^{2} \operatorname{coth}|\beta|^{2}+2|\beta|^{2} \cos 2 \theta+1}{2|\beta|^{2} \operatorname{coth}|\beta|^{2}-2|\beta|^{2} \cos 2 \theta+1}}\left(\alpha_{o p t}^{2}\right)^{(o)}
$$

Thus by using even coherent light is used, under the limit $1 \ll|\beta|^{2} \ll \alpha^{2}$ and $\theta_{2}=\pi / 2$, yields

$$
\left(\alpha_{o p t}^{2}\right)^{(e v)}=\frac{\left(\alpha_{o p t}^{2}\right)^{(o)}}{2|\beta|}
$$

This result, which is also true for odd coherent states, allows an alternative way to decrease the optimal input power, i.e., an alternative way to increase the sensitivity of the interferometer. We have, therefore, given a positive answer to the question originally posed. The question whether this new way might be experimentally achievable or not is left open, depending on the actual physical generation of the even and odd coherent states.

We wish now to consider more general situations: namely for case (i) $\xi$ is arbitrary and for case (ii) $\beta$ is arbitrary and in both situations $\alpha$ is real. $(\Delta z)^{2}$ is a function of such variables and we can look for its minimization with respect to $\alpha^{2}$. This results for case (i) in $\theta_{1}=0$ and $\left(\alpha_{o p t}^{2}\right)^{(s q)}$ function of $\mathrm{r}$ and for case (ii) in $\theta_{2}=\pi / 2$ and $\left(\alpha_{o p t}^{2}\right)^{(e v)},\left(\alpha_{o p t}^{2}\right)^{(o d d)}$ functions of $|\beta|$. In each case, $\alpha_{o p t}^{2}$ as function of the respective independent variable, is given through a solution of the following equation in $\xi$

$$
\Gamma_{1} \xi^{3}-\Gamma_{2} \xi-2 \Gamma_{3}\left(1+\Gamma_{2}\right)=0
$$


in which

$$
\xi=\frac{\alpha_{o p t}^{2}}{\left(\alpha_{o p t}^{2}\right)^{(o)}}-\Gamma_{3}
$$

and

$$
\begin{aligned}
& \Gamma_{1}=e^{2 r} \\
& \Gamma_{2}=e^{-2 r} \\
& \Gamma_{3}=\frac{\sinh ^{2} r}{\left(\alpha_{o p t}^{2}\right)^{(o)}}
\end{aligned}
$$

for the squeezed vacuum, and

$$
\begin{aligned}
& \Gamma_{1}=2|\beta|^{2} \tanh |\beta|^{2}-2|\beta|^{2} \cos 2 \theta_{2}+1 \\
& \Gamma_{2}=2|\beta|^{2} \tanh |\beta|^{2}+2|\beta|^{2} \cos 2 \theta_{2}+1 \\
& \Gamma_{3}=\frac{|\beta|^{2} \tanh |\beta|^{2}}{\left(\alpha_{o p t}^{2}\right)^{(o)}}
\end{aligned}
$$

for the even coherent states. For the quantities relative to odd coherent states same formulas apply with $\operatorname{coth}|\beta|^{2}$ in place of $\tanh |\beta|^{2}$.

Equation (34) has three roots, two of them complex and the physical one has the following form

$$
\begin{aligned}
\alpha^{\prime} \equiv \frac{\alpha_{o p t}^{2}}{\left(\alpha_{o p t}^{2}\right)^{o}} & =\frac{3^{\frac{3}{2}} \sqrt{\Gamma_{1}} \Gamma_{4} \Gamma_{3}+9 \Gamma_{2}+\Gamma_{4}^{2}}{3^{\frac{3}{2}} \sqrt{\Gamma_{1}} \Gamma_{4}} \\
\Gamma_{4} & =\left(9 \Gamma_{3} \sqrt{\Gamma_{1}}\left(1+\Gamma_{2}\right)+\sqrt{81 \Gamma_{1} \Gamma_{3}^{2}\left(1+\Gamma_{2}\right)^{2}-\Gamma_{2}^{3}}\right)^{\frac{1}{3}}
\end{aligned}
$$

Fig.(2), illustrates case (i) and $\alpha^{\prime}$ is plotted versus the squeezing parameter $\mathrm{r}$. For $\mathrm{r}=0$ we have the situation in which the only vacuum fluctuations enter from $P_{2}$ and in this case $\alpha^{\prime}=1$. For large values of $\mathrm{r}$, we have Caves result [2], i.e., the optimal value of the input coherent field through $P_{1}$ can be decreased to a large amount.

With the same spirit, in Fig.(3a), we have plotted $\left(\alpha^{\prime}\right)_{e v}^{-1}$ versus $|\beta|$ and $\theta_{2}$ and in Fig.(3b) this quantity versus $|\beta|$ at $\theta_{2}=\pi / 2$, which is the value for all the local minima, when even coherent light enters from port 2 . The analogous graphical analysis for odd coherent states is shown in Figs.(4a) and (4b).

Such figures illustrate how the optimal values of the input coherent field in $P_{1}$ can be reduced considerably and allows us to predict an application of even or odd coherent fields.

In conclusion it has been shown that such states might offer a new technique to reduce the optimal power of the input coherent laser and for a better sensitivity of the interferometer. 


\section{Acknowledgements}

M.A.M. and V.I.M. wish to thank the Dipartimento di Scienze Fisiche, of the Universita' di Napoli and I.N.F.N. for the kind hospitality. The research of N.A.A. was supported by the International Center for Theoretical Physics Programme for Research and Training in Italian laboratories, Trieste, Italy.

\section{References}

1. see for example, "The detection of gravitational waves", edited by D.G. Blair (Cambridge University Press, Cambridge, 1991), and the references there in.

2. C.M. Caves, Phys. Rev. A 23, (1981) 1693; Phys. Rev. Lett. 45, 75 (1980).

3. R.J. Glauber, Phys. Rev. Lett. 10, 84 (1963).

4. D. Stoler, Phys. Rev. D 42309 (1971); J.N Hollenhorst Phys. Rev. D 19, 1669 (1979); E.Y. Lu, Lett. Nuovo Cim. 3, 585 (1972); D.P. Bertrand, K. Moy. E.A. Mishkin, Phys. Rev D 4, 1909 (1971); H.P. Yuen, Phys. Rev A 13, 2226 (1976); D.F. Walls Nature 306, 141 (1983).

5. V. V. Dodonov, I. A. Malkin, and V. I. Man'ko, Physica 72, 597 (1974).

6. J. Gea-Banacloche, Phys. Rev. A 44, 5913 (1991).

7. C. C. Gerry and E. E. Hach III, Phys. Lett. A 74, 185 (1993)

8. V. Buzek, A. Vidiella-Barranco and P.L. Knight, Phys. Rev. A 45, 6570 (1992).

9. J. Jansky and A.V. Vinogardov, Phys. Rev. Lett. 64, 2771 (1990).

10. J. Perina, Quantum Statistics of Linear and Non-Linear Optical Phenomena (Reidel, Dordrecht, 1984) p. 78.

11. C.C. Gerry Optics Commun. 91, 247 (1992).

12. S. Solimeno, F. Barone, C.de Lisio, L.Di Fiore, L. Milano, and G. Russo, Phys. Rev. A 43, (1991) 6227. 


\section{Figure Captions}

Fig.(1). Schemetic of the simple Michelson interferometer $a_{1}, a_{2}$ and $, c_{1}, c_{2}$ are respectively the input and output fields, while $b_{1}, b_{2}$ stand for the fields incident on mirrors, $M_{1}$ and $M_{2}$.

Fig.(2). Relative value $\left(\alpha^{\prime}\right)_{s q}$ of the optimal laser intensity in the presence and of the squeezed vacuum mode $a_{2}$, versus the squeezing parameter r.

Fig.(3a). Three dimensional plot of the relative value $\left(\alpha^{\prime}\right)_{e v}^{-1}$ of the optimal laser intensity in the presence of even coherent states versus $|\beta|$ and $\theta_{2}$.

Fig.(3b). $\left(\alpha^{\prime}\right)_{e v}$ versus $|\beta|$ for $\theta_{2}=\pi / 2$.

Fig.(4a) Three dimensional plot of the relative value $\left(\alpha^{\prime}\right)_{\text {odd }}^{-1}$ of the optimal laser intensity in the presence and in the absence of odd coherent states versus $|\beta|$ and $\theta_{2}$.

Fig.(4b). $\left(\alpha^{\prime}\right)_{\text {odd }}$ versus $|\beta|$ for $\theta_{2}=\pi / 2$. 